\title{
Rare case of disseminated fusariosis in a young patient with graft vs. host disease following an allogeneic transplant
}

\author{
ALINA TANASE $^{1 *}$, ANCA COLITA $^{1^{*}}$, GABRIEL IANOSI $^{2 *}$, DANIELA NEAGOE $^{3 *}$, \\ DACIANA ELENA BRANISTEANU ${ }^{4 *}$, DANIELA CALINA ${ }^{5 *}$, ANCA OANA DOCEA $^{6 *}$, \\ ARISTIDIS TSATSAKIS ${ }^{7^{*}}$ and SIMONA LAURA IANOSI ${ }^{8^{*}}$
}

\author{
${ }^{1}$ Bone Marrow Transplant Center, Fundeni Clinical Institute, 022328 Bucharest; \\ Departments of ${ }^{2}$ Surgery and ${ }^{3}$ Internal Medicine, University of Medicine and Pharmacy of Craiova, 200349 Craiova; \\ ${ }^{4}$ Department of Dermatology, University of Medicine and Pharmacy 'Gr. T. Popa', 700115 Iasi; Departments of \\ ${ }^{5}$ Clinical Pharmacy and ${ }^{6}$ Toxicology, University of Medicine and Pharmacy of Craiova, 200349 Craiova, Romania; \\ ${ }^{7}$ Laboratory of Toxicology, Medical School, University of Crete, Voutes, Heraklion 71409, Greece; \\ ${ }^{8}$ Department of Dermatology, University of Medicine and Pharmacy of Craiova, 200349 Craiova, Romania
}

Received December 12, 2015; Accepted March 23, 2016

DOI: $10.3892 /$ etm.2016.3562

\begin{abstract}
Fusarium infection is a severe fungal infection caused by fungi of the genus Fusarium. It most commonly occurs in immunocompromised patients with malignant hematological comorbidities or secondary to hematopoietic stem cell transplant. The classical route of contamination is through inhalation but infection may also occur through contiguity with a skin lesion. This report describes the case of a 24-year-old woman who developed graft-vs.-host disease (GVHD) at 220 days after receiving an allogeneic stem cell transplant from a sibling donor for Hodgkin disease. On day 330 after transplant the patient presented with fever and several painful subcutaneous, tender, red nodules with ulcerative and necrotic features on the pelvic region and right leg, extensive glass infiltrative lesions in the lungs and pansinusitis; however, the patient did not have onychomycosis. Following skin biopsy, culture of cutaneous lesions, computed tomography (CT) scanning of the lungs and CT scanning and magnetic resonance imaging of facial sinuses the patient was diagnosed with disseminated Fusarium species infection. Despite intensive treatment with voriconazole, the patient succumbed with respiratory insufficiency on day 400 after transplant. This case is noteworthy because the patient did not have any additional risk associated with the allogeneic transplant; there was no
\end{abstract}

Correspondence to: Professor Daciana Elena Branisteanu, Department of Dermatology, University of Medicine and Pharmacy 'Gr. T. Popa', 16 Universitatii Street, 700115 Iasi, Romania

E-mail: debranisteanu@yahoo.com

*Contributed equally

Key words: fusariosis, immunosuppression, allogeneic stem cell transplant transplant mismatch, no severe neutropenia and no prior clinical signs of onychomycosis. The association of skin lesions with GVHD lesions increased the initial immunosuppression and delayed diagnosis.

\section{Introduction}

Fusarium is a genus of filamentous fungi spread in cereals, fruits and vegetables, water and air, and even in the soil in temperate climates. It affects human health when infested foods or water are consumed (1). Almost all Fusarium species are harmless, although some species (Fusarium solani, Fusarium oxysporum, Fusarium chlamydosporum and Fusarium moniliforme) produce mycotoxins, such as fumonisins and trichothecenes, causing opportunistic infections in healthy humans or hematogenic spreading to immunocompromised individuals, with very poor prognosis (2).

Contamination with Fusarium saprophytic species can cause infections in humans that are localized or disseminated throughout the body $(2,3)$. The most common clinical aspects of Fusarium infection in immunocompetent patients may occur in nails (onychomycosis), in skin, caused particularly by trauma or burns (subcutaneous tender nodules such as nodous erythema, ecthyma-like lesions and cellulitis), or in viscera (cornea, lungs, heart or joints) (4).

Disseminated Fusarium infections cause significant morbidity and mortality in immunocompromised patients, being the second most frequent fungal infection after aspergillosis (1). Infection is characterized by cutaneous nodules, positive fungemia in $40 \%$ of cases and visceral involvement, particularly in lungs and sinuses, with a high rate of mortality (5). Studies have shown that among patients who have received a hematopoietic stem cell transplant (HSCT) the frequency of fusariosis ranges between 4.21-5.0 cases per 1,000 in human leukocyte antigen (HLA)-matched related transplant recipients to 20.19 cases per 1,000 in HLA-mismatched transplant recipients $(1,3)$. 
There are few cases presented in the literature of disseminated fusariosis infection in patients who have received an allogeneic stem cell transplant (4). The present report describes a rare clinical case of a female who developed graft-vs.-host disease (GVHD) following an allogeneic stem cell transplant for Hodgkin disease and succumbed after contracting disseminated Fusarium infection. Another particularity of this case was that the patient did not present clinical onychomycosis.

\section{Case report}

In February 2009, a 24-year old women received an allogeneic stem cell transplant from a sibling donor for Hodgkin's disease (chemosensitive relapse following autologous stem cell transplant). The patient achieved a complete remission, with negative positron emission tomography-computed tomography (PET-CT) findings at 100 days after transplant, with full donor chimerism. On day 220 after transplant the patient developed moderate GVHD (skin 2, mouth 1, eye 1) (6) with atrophic and sclerodermic skin on the thighs. Cutaneous histological examination revealed atrophic epidermis, intense collagenous sclerosis in the papillary dermis, basal cell vacuolization and apoptotic keratinocytes that confirmed the GVHD diagnosis (Fig. 1). Immunosuppressive treatment was initiated with $1 \mathrm{mg} / \mathrm{kg} /$ day methylprednisolone, and prophylactic treatment with $400 \mathrm{mg} /$ day fluconasol and 1,000 mg/day acyclovir. Despite this treatment, sclerodermic features of the skin spread, involving progressively the lower abdomen, thighs and forearms. This was considered as progressive GVHD and the immunosuppressive therapy was increased, initially with $2 \mathrm{mg}$ /day tacrolimus, and then with $2 \mathrm{~g} /$ day mycofenolate mofetil.

On day 330 after transplant the patient presented with fever and several painful subcutaneous, tender, red nodules that quickly became ulcerative, in addition to necrotic features on the pelvic region and right leg localized on atrophic and sclerodermic skin (Fig. 2). The skin biopsy from one of these lesions at the level of reticular dermis revealed intravascular embolus containing fungal hyphae elements stained periodic acid-Schiff positive and lymphocytic infiltrate around the vessel wall (Fig. 3).

When wide spectrum antibiotic treatments were administered, the patient's condition was aggravated, with severe acute respiratory and kidney insufficiency that required ventilatory assistance and peritoneal dialysis (single sessions at 2-3-day intervals).

CT scanning of the lungs revealed extensive glass infiltrative lesions (Fig. 4) and CT scanning and magnetic resonance imaging of the facial sinuses showed pansinusitis (Figs. 5 and 6).

Two swabs from skin lesions were performed and cultured on Sabouraud dextrose agar, sheep blood agar and cystine lactose electrolyte deficient agar. After 48 h, rapidly growing fluffy colonies were observed, with a distinct rose-like surface and reverse-side pigmentation (4-6 days; Fig. 7). Microscopic examination performed by culture, with adhesive tape (lactophenol smear), revealed hyaline filamentous moulds, producing conidia (microconidia and macroconidia) in clusters. Of the hyaline filamentous molds, Fusarium spp. is unique in producing microconidia and macroconidia (7). The key to the identification of Fusarium spp. was the observation of long, sickle-form, multicellular macroconidia, separated

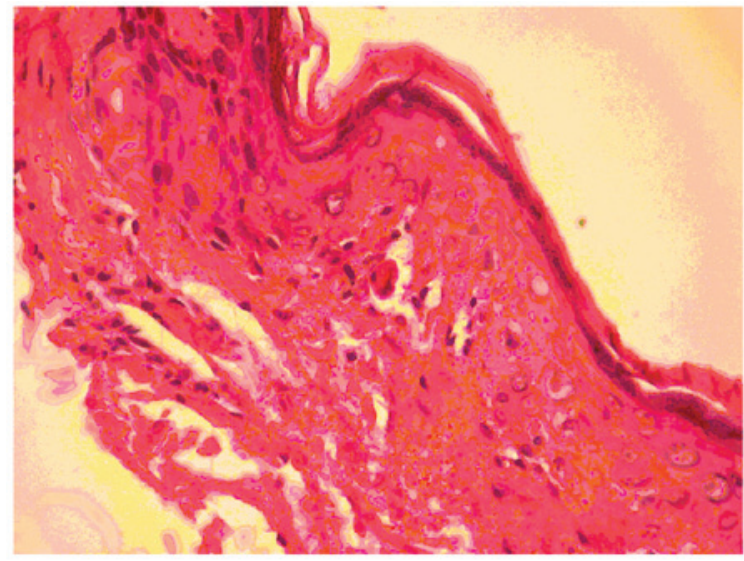

Figure 1. Graft-vs.-host disease: Atrophic epidermis, intense collagenous sclerosis in the papillary dermis, basal cell vacuolization and apoptotic keratinocytes. Hematoxylin and eosin staining, magnification, x10.

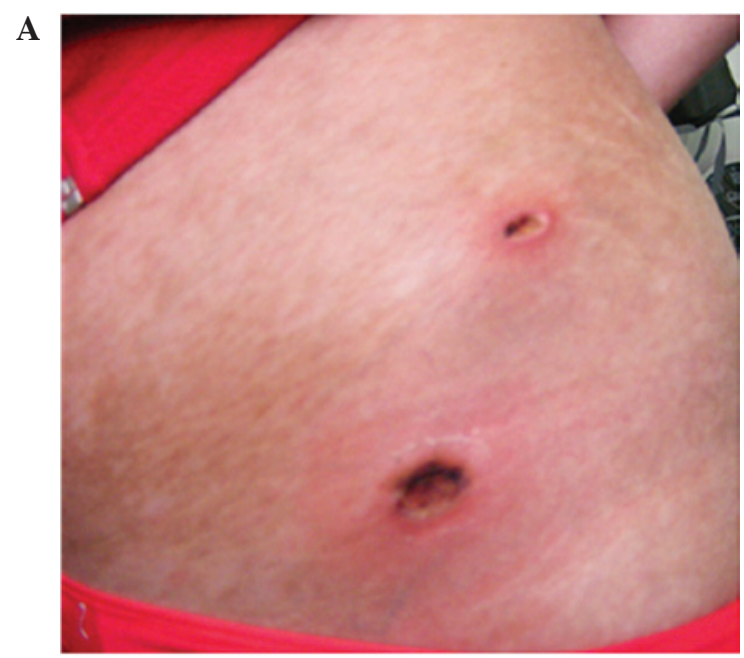

$\mathbf{B}$

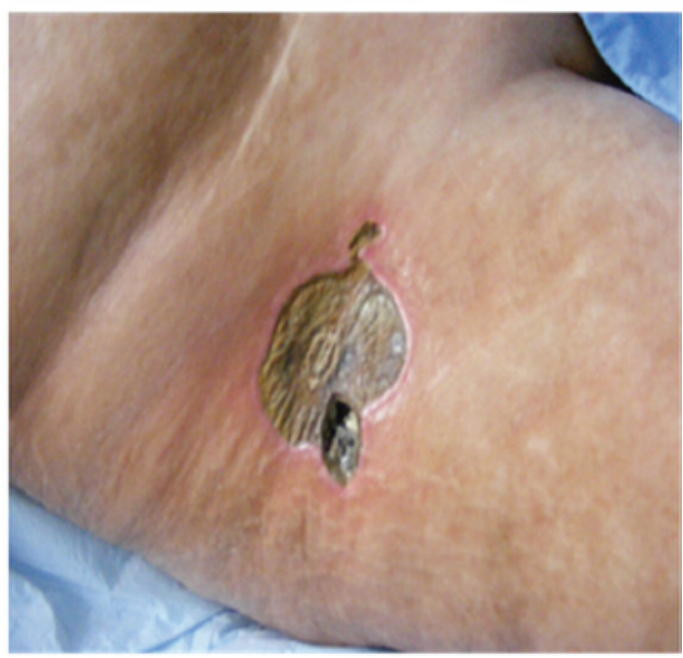

Figure 2. Ulcerative and necrotic nodules on (A) the pelvic region (A) and (B) the right leg.

by transverse septa. These macroconidia may be described as 'canoes' or 'boats' (Fig. 8). Following the examination of the culture and smear, it was concluded that the hyaline molds were medically significant, producing conidia in clusters, and comprised a Fusarium species. 


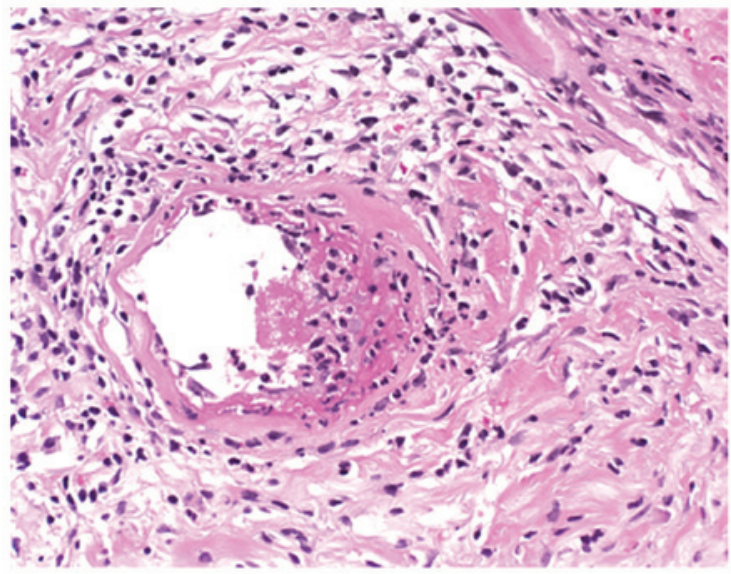

Figure 3. Intravascular embolus containing fungal hyphae elements stained periodic acid-Schiff (PAS) positive in the reticular dermis and lymphocytic infiltrate around the vessel wall. PAS staining; magnification, $\mathrm{x} 10$.

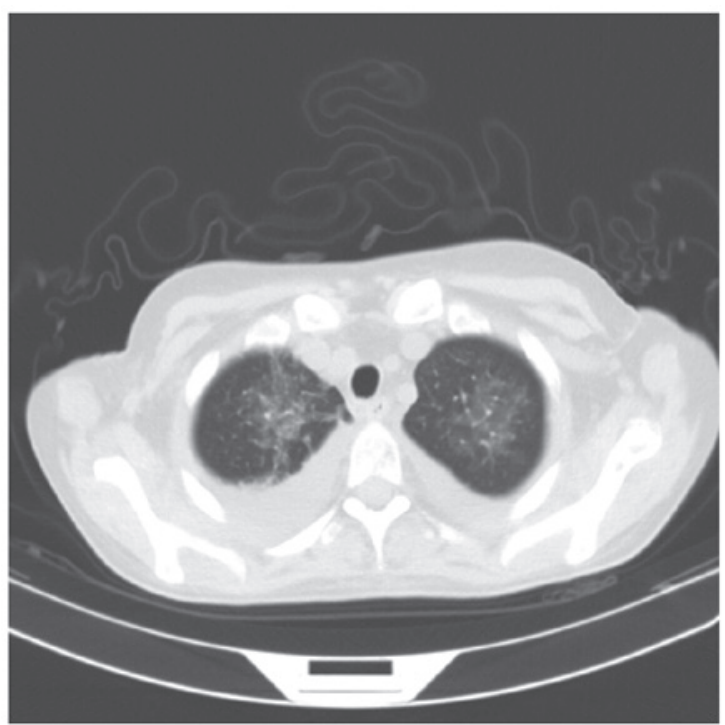

Figure 4. Lung computed tomography scan showing extensive glass infiltrative lesions.

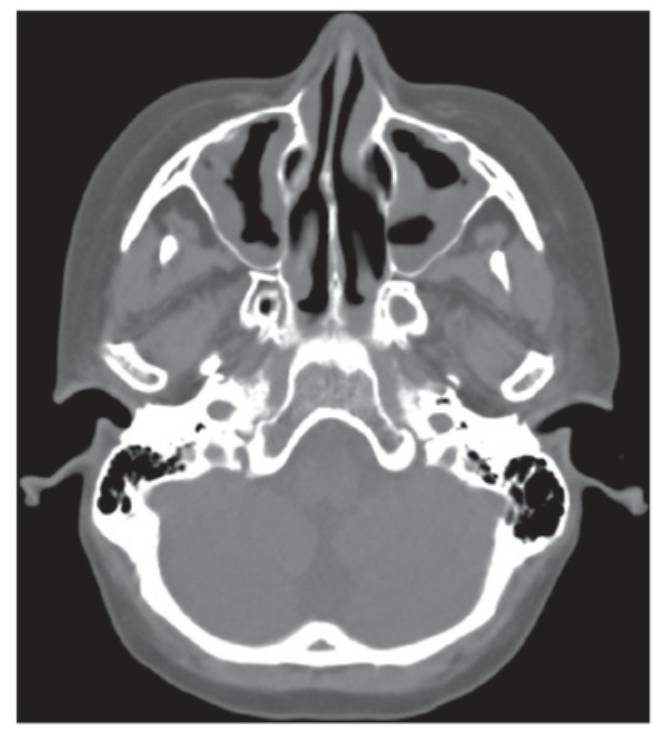

Figure 5. Computed tomography scan of the sinuses indicating pansinusitis.

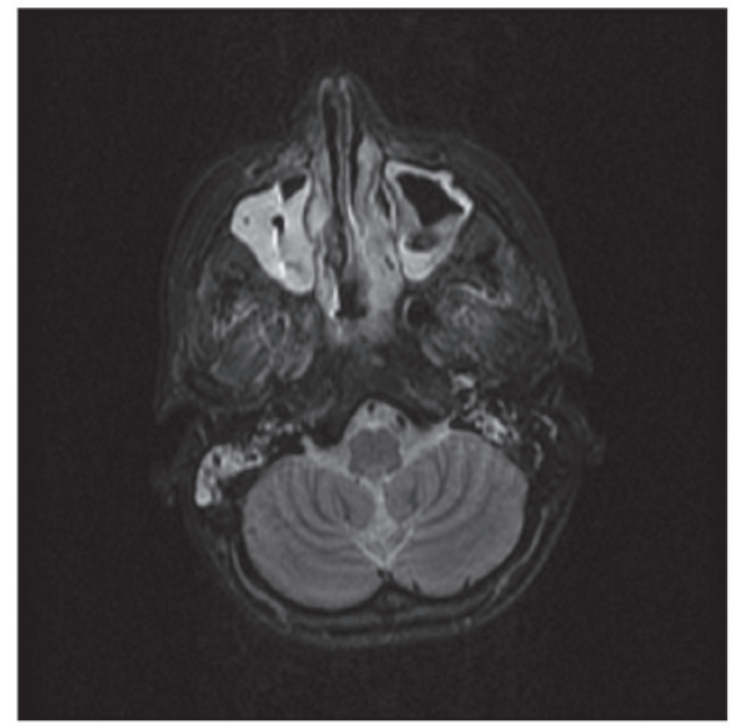

Figure 6. Magnetic resonance imaging of the sinuses indicating pansinusitis

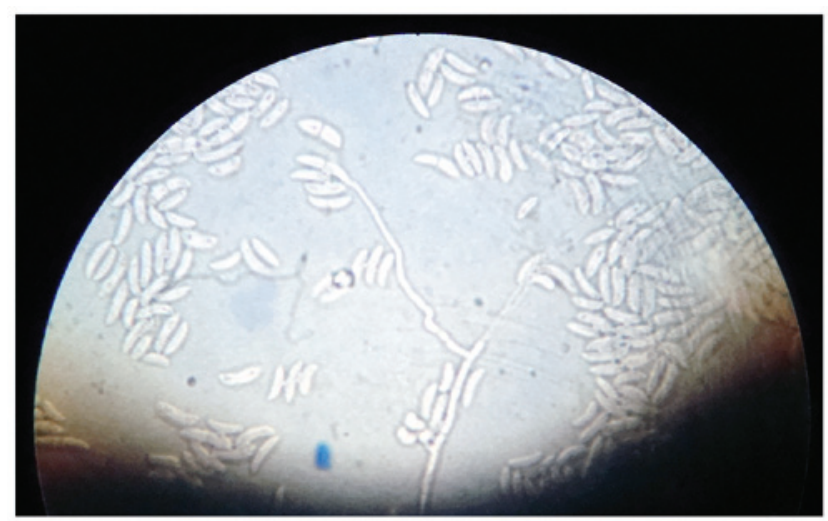

Figure 7. Fluffy colonies, with a distinct rose-like surface and reverse-side pigmentation (culture on Sabouraud dextrose agar).

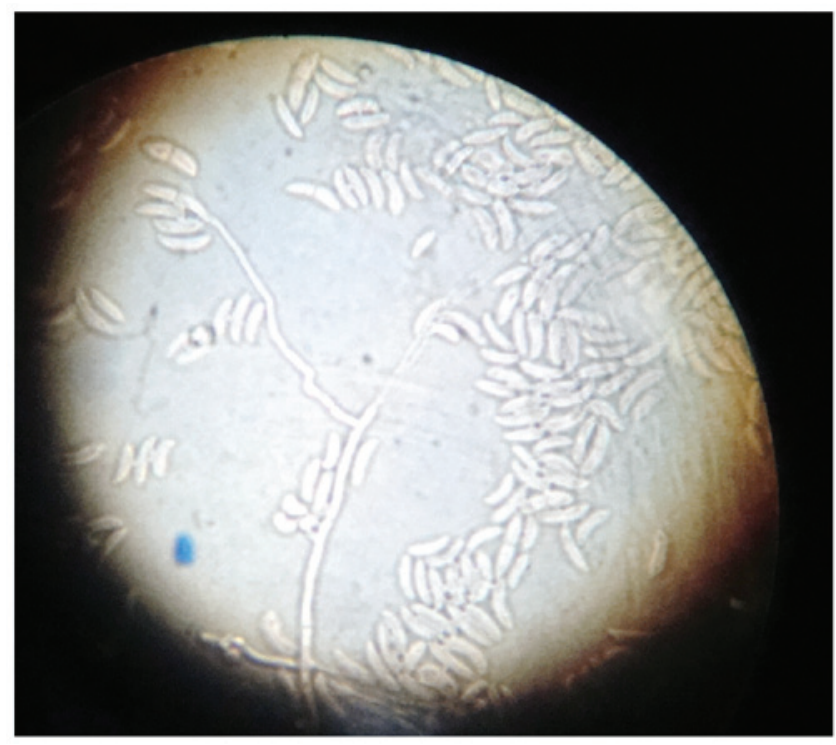

Figure 8. Microscopic examination performed by culture using hyaline filamentous moulds showed the production of conidia (microconididia and macroconidia) in clusters. Long, sickle-form, multicellular macroconidia separated by transverse septa are described as 'canoes' or 'boats'. 
Laboratory findings were as follows: Hemoglobin level, $8,9 \mathrm{~g} / \mathrm{dl}$; leucopenia $\left(3,000 / \mathrm{mm}^{3}\right) ;$ mild neutropenia $\left(1,400 / \mathrm{mm}^{3}\right) ;$ creatinine, $1.3 \mathrm{mg} / \mathrm{dl}$; urea, $47 \mathrm{mg} / \mathrm{dl}$; alkaline phosphatase, $101 \mathrm{U} / 1$; lactate dehydrogenase, $293 \mathrm{U} / 1$; C-reactive protein, $92 \mathrm{mg} / \mathrm{l}$.

Repeated hemocultures were performed during fever outbreaks without fungus identification. Correlating the investigations with clinical aspects the diagnosis of Fusarium species infection was established in this patient with GHVD following allogeneic transplant.

Under these conditions, voriconazole therapy was initiated while the GHVD immunosuppression therapy was progressively reduced. While the treatment with intravenous voriconazole was ongoing, which comprised a $6-\mathrm{mg} / \mathrm{kg}$ loading dose every $12 \mathrm{~h}$ for the first $24 \mathrm{~h}$, and a $4-\mathrm{mg} / \mathrm{kg}$ maintenance dose every $12 \mathrm{~h}$ thereafter, the cutaneous lesions initially improved, but the patient's clinical condition deteriorated continuously. Despite continued treatment with voriconazole, the patient succumbed with respiratory insufficiency due to the overwhelming infection 400 days after receiving the transplant.

Written informed consent was obtained from the patient's family prior to publication of the present study.

\section{Discussion}

There is a very high risk of contamination with Fusarium species for patients who have received an allogeneic bone marrow transplant, either early after transplant (when engraftment is delayed), or later, due to deep immunosuppression secondary to prolonged treatment with cortisone for acute or chronic GVHD (2-4,8). Fusarium infections in solid-organ transplant recipients have a localized character and a better prognosis compared with those developed after hematologic malignancies and bone marrow transplant (4).

Current studies have shown an increase in the frequency of fungal infections caused by unusual opportunistic fungi such as Fusarium species (9). The notable increase in organ-transplant procedures and newer aggressive approaches to immunosuppression are largely responsible for this shift.

It is known that $>90 \%$ of cases of invasive fusariosis are linked to immunosuppression and this appears particularly in patients with hematologic malignancies and neutropenia $(2,4)$. Fusariosis occurs mostly in patients who have received a mismatched or unrelated transplant (8). Nucci et al observed a tri-modal distribution for invasive fusariosis in patients who received an allogeneic $\mathrm{HSCT}$, with a maximum incidence prior to engraftment, and at $\sim 62$ days and 11 years after transplantation (1). Persistent neutropenia represents the most important prognostic variable. In the management of Fusarium infections, neutrophils play an important role. Once Fusarium species enter the body, neutrophils attach to the Fusarium hyphae and destroy them extracellularly through oxidative cytotoxic mechanisms $(10,11)$. This mechanism is supported by the fact that patients with profound and prolonged neutropenia and associated fusariosis show 100\% mortality even under substantial antifungal therapy (12). There are two factors involved in the pathogenesis of fusariosis: one is the Fusarium strain's virulence, and the other is the host's immune status where immunosuppression plays an overwhelming role in the disease. The aggressiveness of Fusarium species is caused by several mycotoxins that they produce $(13,14)$. Some of these mycotoxins can cause leukopenia that prolongs chemotherapy-induced bone marrow suppression (15). Fusarium species have angiotropic and angioinvasive effects and can produce hemorrhagic infarction, followed by decreased tissue perfusion and tissue necrosis similar to that observed with Aspergillus species and Zygomycetes species $(4,8,10)$.

Patients who have received a transplant from a matched unrelated donor or missmatch related donor and/or are receiving therapy for extensive chronic GVHD may develop severe T-cell-mediated immunodeficiency, which is a potential risk for Fusarium infections $(2,12)$. This population of patients develops very late fusariosis. Patients with hematologic cancer under glucocorticoids therapy showed $70 \%$ mortality for fusariosis compared with $33 \%$ for those not receiving glucocorticoids (11).

Histopathological diagnosis of fusariosis is made by identifying fungi with septate hyphae and acute angle-branching. These histological changes are difficult to distinguish from those induced by the common fungus Aspergillus or of those produced by the less common, relatively harmless fungus Pseudallescheria boydii (16). For a more precise identification of Fusarium species, blood or tissue cultures should be performed. The results of these cultures can indicate a diagnosis of proven or probable fusariosis. A diagnosis of probable fusariosis is made for patients with clinical manifestations if the Fusarium species are isolated and identified in respiratory tract secretions in the absence of other pathogens or if there is a positive culture from skin lesions but hyphae are not histopathologically identified. A diagnosis of proven fusariosis can be made if Fusarium species are identified in blood cultures or in cultures obtained from sterile sites taken from patients with clinical signs of fungal infection or if hyphae and the Fusarium species are identified together in the same tissue (17).

Pathogenic fungi can be differentiated using new molecular techniques such as in situ hybridization against ribosomal RNA sequences (18) and polymerase chain reactions (19-21). The disadvantages of these techniques are their high cost and low availability. However, certain studies have shown that these two molecular techniques have promising results $(18,21)$. In the case of histologically identical fungi, it is very important to correctly identify them as Fusarium and Pseudallescheria are more resistant to antifungal pharmacotherapy than is Aspergillus (22).

In cases of severe fusariosis, the therapeutic alternatives are reduced. Once it is diagnosed, invasive fusariosis requires immediate therapy because of its rapidly evolvement and high mortality rate. An effective treatment for invasive fusariosis has not yet been identified, but good results have been achieved with high doses of amphotericin B, particularly in lipid formulation and with antifungals from the triazole class such as posaconazole and voriconazole. Voriconazole is indicated as the first line therapy for fusariosis (23-25).

In the management of Fusarium infections, preventive measures are more important than antifungal therapy, firstly because the latter has a low rate of success in HSCT recipients and secondly because Fusarium species are usually resistant to this therapy. In this regard, the clinician must carefully examine skin and tissue lesions, particularly onychomycoses, as these are important sites of contamination with Fusarium 
species $(26,27)$. The objectives in future therapies for fusariosis are suggested to be reduction of the duration of neutropenia, reduction of immunosuppressive therapy and the administration of novel antifungal agents such as posaconazole and voriconazole $(28,29)$.

In conclusion, it is important to underline that a patient receiving an allogeneic stem cell transplant can be at risk for invasive fusariosis and overlapping GVHD lesions with Fusarium skin lesions may delay diagnosis and treatment.

\section{Acknowledgements}

This study was partially supported by the Sectoral Operational Programme 'Human Resources Development', financed from the European Social Fund and by the Romanian Government under the contract number POSDRU/89/1.5/S/64109.

\section{References}

1. Nucci M, Marr KA, Queiroz-Telles F, Martins CA, Trabasso P, Costa S, Voltarelli JC, Colombo AL, Imhof A, Pasquini R, et al: Fusarium infection in hematopoietic stem cell transplant recipients. Clin Infect Dis 38: 1237-1242, 2004.

2. Marr KA, Carter RA, Crippa F, Wald A and Corey L: Epidemiology and outcome of mould infections in hematopoietic stem cell transplant recipients. Clin Infect Dis 34: 909-917, 2002

3. Galimberti R, Torre AC, Baztán MC and Rodriguez-Chiappetta F: Emerging systemic fungal infections, Clin Dermatol 30: 633-650, 2012.

4. Campo M, Lewis RE and Kontoyiannis DP: Invasive fusariosis in patients with hematologic malignancies at a cancer center: 1998-2009. J Infect 60: 331-337, 2010.

5. Stanzani M, Tumietto F, Vianelli N and Baccarani M: Update on the treatment of disseminated fusariosis: Focus on voriconazole. Ther Clin Risk Manag 3: 1165-1173, 2007.

6. Filipovich AH, Weisdorf D, Pavletic S, Socie G, Wingard JR, Lee SJ, Martin P, Chien J, Przepiorka D, Couriel D, et al: National Institutes of Health consensus development project on criteria for clinical trials in chronic graft-versus-host disease: I. Diagnosis and staging working group report. Biol Blood Marrow Transplant 11: 945-956, 2005.

7. Girmenia C, Pagano L, Corvatta L, Mele L, del Favero A and Martino P: The epidemiology of fusariosis in patients with haematological diseases. Gimema Infection Programme. Br J Haematol 111: 272-276, 2000.

8. Minor RL Jr,Pfaller MA, Gingrich RD and Burns LJ: Disseminated Fusarium infections in patients following bone marrow transplantation. Bone Marrow Transplant 4: 653-658, 1989.

9. Jain A, Jain S and Rawat S: Emerging fungal infections among children: A review on its clinical manifestations, diagnosis, and prevention. J Pharm Bioallied Sci 2: 314-320, 2010.

10. Clemons KV, Calich VL, Burger E, Filler SG, Grazziutti M, Murphy J, Roilides E, Campa A, Dias MR, Edwards JE Jr, et al: Pathogenesis I: Interactions of host cells and fungi. Med Mycol 38 (Suppl 1): S99-S111, 2000.

11. Kontoyiannis DP, Bodey GP, Hanna H, Hachem R, Boktour M, Girgaway E, Mardani M and Raad II: Outcome determinants of fusariosis in a tertiary care cancer center: The impact of neutrophil recovery. Leuk Lymphoma 45: 139-141, 2004.

12. Nucci M, Anaissie EJ, Queiroz-Telles F, Martins CA, Trabasso P, Solza C, Mangini C, Simões BP, Colombo AL, Vaz J, et al: Outcome predictors of 84 patients with hematologic malignancies and Fusarium infection. Cancer 98: 315-319, 2003.

13. Short DP, O'Donnell K, Zhang N, Juba JH and Geiser DM: Widespread occurrence of diverse human pathogenic types of the fungus Fusarium detected in plumbing drains. J Clin Microbiol 49: 4264-4272, 2011.
14. Pitt JI: Toxigenic fungi: Which are important? Med Mycol 38 (Suppl 1): S17-S22, 2000.

15. Perfect JR: The impact of the host on fungal infections. Am J Med 125 (Suppl): S39-S51, 2012.

16. Muhammed M, Coleman JJ, Carneiro HA and Mylonakis E: The challenge of managing fusariosis. Virulence 2: 91-96, 2011.

17. De Pauw B, Walsh TJ, Donnelly JP, Stevens DA, Edwards JE, Calandra T, Pappas PG, Maertens J, Lortholary O, Kauffman CA, et al; European Organization for Research and Treatment of Cancer/Invasive Fungal Infections Cooperative Group; National Institute of Allergy and Infectious Diseases Mycoses Study Group (EORTC/MSG) Consensus Group: Revised definitions of invasive fungal disease from the European Organization for Research and Treatment of Cancer/Invasive Fungal Infections Cooperative group and the National Institute of Allergy and Infectious Diseases Mycoses Study Group (EORTC/MSG) Consensus Group. Clin Infect Dis 46: 1813-1821, 2008.

18. Hayden RT, Isotalo PA, Parrett T, Wolk DM, Qian X, Roberts GD and Lloyd RV: In situ hybridization for the differentiation of Aspergillus, Fusarium, and Pseudallescheria species in tissue section. Diagn Mo Pathol 12: 21-26, 2003.

19. Leal SM Jr, Vareechon C, Cowden S, Cobb BA, Latgé JP, Momany $M$ and Pearlman E: Fungal antioxidant pathways promote survival against neutrophils during infection. J Clin Invest 122: 2482-2498, 2012.

20. Walsh TJ, Francesconi A, Kasai M and Chanock SJ: PCR and single-strand conformational polymorphism for recognition of medically important opportunistic fungi. J Clin Microbiol 33: 3216-3220, 1995.

21. Hennequin C, Abachin E, Symoens F, Lavarde V, Reboux G, Nolard $\mathrm{N}$ and Berche P: Identification of Fusarium species involved in human infections by $28 \mathrm{~S}$ rRNA gene sequencing. J Clin Microbiol 37: 3586-3589, 1999.

22. Pfaller MA, Messer SA, Hollis RJ and Jones RN; SENTRY Participants Group: Antifungal activities of posaconazole, ravuconazole, and voriconazole compared to those of itraconazole and amphotericin B tested against 239 clinical isolates of Aspergillus spp. and other filamentous fungi: Report from the SENTRY antimicrobial surveillance program, 2000. Antimicrob Agents Chemother 46: 1032-1037, 2002.

23. Nucci $M$ and Anaissie E: Cutaneous infection by Fusarium species in healthy and immunocompromised hosts: Implications for diagnosis and management. Clin Infect Dis 35: 909-920, 2002.

24. Bodey GP, Boktour M, Mays S, Duvic M, Kontoyiannis D, Hachem R and Raad I: Skin lesions associated with Fusarium infection. J Am Acad Dermatol 47: 659-666, 2002.

25. Segal BH, Almyroudis NG, Battiwalla M, Herbrecht R, Perfect JR, Walsh TJ and Wingard JR: Prevention and early treatment of invasive fungal infection in patients with cancer and neutropenia and in stem cell transplant recipients in the era of newer broad-spectrum antifungal agents and diagnostics adjuncts. Clin Infect Dis 44: 402-409, 2007.

26. Anaissie EJ, Kuchar RT, Rex JH, Francesconi A, Kasai M, Müller FM, Lozano-Chiu M, Summerbell RC, Dignani MC, Chanock SJ and Walsh TJ: Fusariosis associated with pathogenic Fusarium species colonization of a hospital water system: A new paradigm for the epidemiology of opportunistic mold infections. Clin Infect Dis 33: 1871-1878, 2001.

27. Raad I, Tarrand J, Hanna H, Albitar M, Janssen E, Boktour M, Bodey G, Mardani M, Hachem R, Kontoyiannis D, et al: Epidemiology, molecular mycology, and environmental sources of Fusarium infection in patients with cancer. Infect Control Hosp Epidemiol 23: 532-537, 2002.

28. Dannaoui E, Desnos-Ollivier M, Garcia-Hermoso D, Grenouillet F, Cassaing S, Baixench MT, Bretagne S, Dromer F and Lortholary O; French Mycoses Study Group: Candida spp. with acquired echinocandin resistance, France, 2004-2010. Emerg Infect Dis 18: 86-90, 2012.

29. Ho DY, Lee JD, Rosso F and Montoya JG: Treating disseminated fusariosis: Amphotericin B, voriconazole or both? Mycoses 50: 227-231, 2007. 